\title{
Assessment of exclusive breast feeding practice and associated factors in Mecha district, North west Ethiopia
}

\author{
Tesfa Getanew Woldie ${ }^{1}$, Addisu Workineh Kassa ${ }^{3}$, Melkie Edris ${ }^{2}$ \\ ${ }^{1}$ Public Health Emergency Management, Bahir Dar, Ethiopia \\ ${ }^{2}$ Gondar University, Public Health Institute, Department of Nutrition, Gondar, Ethiopia \\ ${ }^{3}$ Amhara Regional Health Bureau, Bahir Dar, Ethiopia
}

\section{Email addresses:}

tegewo@yahoo.com (T. G. Woldie), analid9800@gmail.com (A. W. Kassa), melkiey2004@gmail.com (M. Edris)

\section{To cite this article:}

Tesfa Getanew Woldie, Addisu Workineh Kassa, Melkie Edris. Assessment of Exclusive Breast Feeding Practice and Associated Factors in Mecha District, North West Ethiopia. Science Journal of Public Health. Vol. 2, No. 4, 2014, pp. 330-336.

doi: $10.11648 /$ j.sjph.20140204.24

\begin{abstract}
Introduction: It is realistic that Exclusive breast feeding can significantly reduce the burden of under-five death. About $41 \%$ of global under five deaths occur in Sub-Saharan Africa (SSA) mainly due to inadequate breastfeeding practices in combination with high levels of disease. Despite the universal practice of breastfeeding, most women do not practice exclusive breast feeding in many developing countries including Ethiopia. Methods: A community-based cross sectional study was conducted. A total of 819 mothers with 6-12 month aged infants were included in the study. All mothers from randomly selected kebeles were included in the study through cluster sampling technique in Mecha District, Amhara Region in April 2012. Structured interview was conducted in data collection. Both bivariate and multivariate logistic regression techniques were used to analyze the data with 95\% CI. Results: The prevalence of exclusive breast feeding (EBF) was $47.13 \%(95 \% \mathrm{CI} ; 43.7 \%$ - 50.56\%). Mothers who reported having 3 and more antenatal visit during pregnancy $(\mathrm{AOR}=1.7 ; 95 \% \mathrm{CI}: 1.09-2.67)$, who got PNC counseling on infant feeding (AOR=2.27; 95\% CI: 1.56-3.30), who initiated breast feeding $(\mathrm{BF})$ immediately after birth with in the first one hour ( $\mathrm{AOR}=2.98 ; 95 \% \mathrm{CI}: 2.11-4.22$ ) and who have adequate knowledge on $\mathrm{BF}(\mathrm{AOR}=2.06 ; 95 \% \mathrm{CI}: 1.47-2.88)$ were more likely to exclusively breastfeed than their counterparts. Mothers from urban areas were less likely to exclusive breast fed than rural mothers (AOR=0.38; 95\% CI: 0.23-0.62). Conclusions: The prevalence of exclusive breast feeding in the study area was below the WHO recommended level $90 \%$. Residence, three and above ANC visit, NC counseling on infant feeding, immediate initiation of breast feeding (BF) within an hour, maternal knowledge of BF were found to be associated with EBF practice. Strengthening ANC and PNC service, educate pregnant mothers on immediate BF initiation, counseling mothers on infant feeding during PNC, prioritizing urban areas for BF promotion in line with newly launched UHEP and other promotional efforts to improve maternal BF knowledge are recommended.
\end{abstract}

Keywords: Exclusive Breastfeeding, Prevalence, Associated Factors

\section{Introduction}

\subsection{Statement of the Problem}

Suboptimal breast feeding, especially lack of exclusive breastfeeding is the major contributor for infant and child mortality. It attributes $45 \%$ of neonatal infectious deaths, $30 \%$ of diarrheal deaths and $18 \%$ of acute respiratory deaths. It is realistic that exclusive breast feeding (EBF) can significantly reduce the burden of under-five death in Africa especially SSA where $41 \%$ of global under five death occur mainly due to inadequate breastfeeding practices in combination with high levels of disease [1], [2].

In Ethiopia nearly 321,000 under five children die each year [3] from which malnutrition is the cause for about $57 \%$ of deaths primarily through the exacerbation of other major causes, such as diarrhoea and pneumonia death from which can be significantly prevented by nutrition interventions such as exclusive breast feeding [4].

Lack of exclusive breastfeeding during the first 6 month of life is the most important risk factors for infant and 
childhood morbidity and mortality including life-long impact like poor school performance, reduced productivity, and impaired intellectual development [5].

Evidence showed infants who lack exclusive breast feeding are more likely to be attacked and died from major causes of infant mortality like diarrhea and pneumonia. Moreover there is 3.2 fold increase risk for SAM in their childhood life time than those who got EBF [6], [7].

EBF for the first 6 months of infant life with continued BF up to 2 years and beyond can avert $13 \%$ to $15 \%$ of under-five deaths as scientific evidences revealed which shows EBF as effective intervention in child mortality reduction. These evidences also recommend the coverage of EBF to reach $90 \%$ to be benefited from the intervention [8], [9].

In spite of all the recognized advantages and efforts deployed to promote EBF, the practice is still much far from the recommended level. It is as low as $36 \%$ globally, 39\% in developing countries and $31 \%$ in SSA among under 6 month infants [6], [10].

In Ethiopia breast feeding is universal but, only $52 \%$ of mothers who have under 6 month infants exclusively breast feed .This figure will declined to $32 \%$ among 4-5 month aged infants according to the 2011 EDHS [11].

This suggests nearly half of under 6 month infants are not getting the benefits of exclusive breast feeding and also the prevalence decreases with increasing age of infants. This evidence clearly shows, the potential public health importance of EBF to reduce the burden of under-five mortality is still untapped in Ethiopia.

Previous study suggested that Mecha District (i.e. study area) is one of the Districts in Amhara region which is food surplus area but children under five are suffering from chronic and acute malnutrition problem. This study indicated as lack of optimal breast feeding is one of the main contributor for malnutrition problem. This evidence tells that studying the practice and associated factors of EBF in the area is sensible to tackle malnutrition problem of children in the area [12].

Therefore this study will determine the prevalence and factors associated with EBF which in turn will have a significant input in the formulation of appropriate strategies.

\subsection{Literature Review}

\subsubsection{Exclusive Breast Feeding}

Exclusive breastfeeding refers to feeding of an infant only breast milk for the first 6 months of life but nothing else even water with the exception of drops or syrups consisting of vitamins, mineral supplements, or drugs [13].

On the basis of evidence from a systematic review of the optimal duration of exclusive breast feeding recommends exclusive breast feeding for the first six months of life of an infant. This recommendation is based on evidence of the importance of good nutrition in the early months of life and of the crucial role that appropriate feeding practices play in achieving optimal health outcomes [9].

Within the first 6 months of life breast feeding is uniquely suited to the infant's nutritional needs. It is also is away to provide a live substance with unparalleled immunological properties that protect against a host of illnesses and diseases thereby decreasing infant morbidity and mortality. It also has important implication of maternal reproductive health benefit [14]. Most importantly EBF eliminates contamination which makes it especially important in resource poor settings [15].

EBF plays a pivotal role in determining the optimal health and development of infants. Feeding of an infant exclusively breast milk for the first 6 months of life is associated with a decreased risk for many early-life diseases and conditions, including otitis media, respiratory tract infection, diarrhea and early childhood obesity [16].

Moreover findings from follow-up researches showed that EBF, as compared with predominant or mixed breastfeeding, reduces the risk of HIV infection and increases the likelihood of HIV-free survival among infants born to HIV-infected mothers [17], [18].

Generally EBF is among level 1 child survival interventions (i.e. sufficient high-quality evidence of benefit, feasible for delivery at high coverage in low-income settings [8].

\subsubsection{Prevalence and Associated Factors of Exclusive Breastfeeding}

Despite the benefits of exclusive breastfeeding, the prevalence and duration globally is much far from the recommended universal coverage. Globally infants in under 6 month of age, only $36 \%$ are exclusively breastfed. It rarely exceeds $30 \%$ in most regions of the developing world given their greater risk of infection and its consequences [19].

The rate of exclusive breast feeding is low in Africa, especially in west and central Africa which is only $20 \%$. Over the past 10 to 15 years exclusive breastfeeding rates have increased in the developing world as a whole and in many countries of Africa and Asia in particular, however, the progress has been modest, from 33\% in 1995 to $37 \%$ in 2008 in the developing world [3], [20].

Different researches in different areas of the world showed as the practice of exclusive breast feeding is low and there are different influencing factors.

A hospital based study from Northern area of Pakistan showed as the prevalence of EBF was $64.8 \%$.It additionally suggested as male infants and not term infants are more likely to be exclusively breast feed than their counter parts [21].

A research conducted in India among mothers who had 6-12 years of age babies reported that the Prevalence of exclusive breastfeeding was $61.5 \%$.This research showed having a male child, maternal age under 30 years, parity of the mother, infant feeding advice, early initiation of breastfeeding as positively associated with exclusive breast feeding [22].

Another study conducted in Sri Lanka among 4-12 infants reported as the median duration of exclusive breastfeeding was four months and the rates of exclusive breastfeeding at four and six months were $61.6 \%$ and $15.5 \%$ respectively. It 
showed that Muslim followers, lower levels of parental education and being an unemployed mother are associated with early cessation of exclusive breastfeeding [23].

A study among infants under six months of age in peninsular Malaysia estimated the prevalence of exclusive breastfeeding as $43.1 \%$. In addition exclusive breastfeeding was positively associated with rural residence, non-working mothers, multifarious mothers, term infants mothers, mothers with husbands who support breastfeeding [24].

A research conducted in Ghana among 0-6 month infants showed that only $51.6 \%$ of infants got EBF which suggested institutional delivery as one of the factor to practice EBF of the mother [25].

A study done in Egypt among mothers who had babies 6-24 months old reported as 95.8\% breastfeeding their babies, whereas only $9.7 \%$ were exclusively breastfeed their infants for 6 months. Antenatal care visit, early breastfeeding initiation after delivery, male infant, and absence of breastfeeding difficulties were the significant predictors associated with higher chance of exclusive breastfeeding [26].

Another study in Nigeria on under 6 month infants found average exclusive breast feeding rate of $16.4 \%$. The study also revealed that the likelihood of exclusively breast feeding was associated with household income, infant age and ANC visit of mother. This study showed as female infants were more likely to be exclusively breastfed than male infants [3].

In Ethiopia breast feeding is common practice, but a large proportion of mothers do not practice optimal breast feeding. Ethiopian demographic health survey (DHS) 2011 estimated $52 \%$ of under 6 month aged infants are exclusive breast feed. Moreover it is estimated at 4- 5 months of age only $32 \%$ of infants were exclusively breast fed. This national survey estimated median duration of any breast feeding and exclusive breast feeding 25 months and 4.2 months respectively. The finding also showed as the duration of any breast feeding and exclusive breast feeding were 32.7 months and 4.6 months respectively in Amhara region [15].

In line with the inadequate EBF coverage of the national estimates, some research findings showed as the prevalence of EBF is lower than the WHO recommended level and also the practice is associated with different factors. For instance a research based on EDHS 2005 data showed that as the EBF prevalence among under 6 month aged infants was only $49 \%$ and it showed maternal marital status, income and age of an infant as predictors of exclusive breast feeding practice of the mothers [27].

Another study which was intended to identify factors related with EBF and dietary diversity of complementary foods, a case study in Amhara region of Ethiopia, revealed Only $19 \%$ of mothers of $0-5 \mathrm{~m}$ aged infants practiced exclusive breast feeding (EBF). From the study factors such as maternal education, trained delivery services and antenatal clinic visits (ANC) were found to be positively associated with EBF practice [28].

The objective of this study was to assess exclusive breast feeding practice and identify factors that influence the practice among mothers with infants aged 6-12 month in Mecha District, Amhara region, North West Ethiopia.

\section{Methodology}

\subsection{Study Area}

This study was conducted in Mecha district at 12 randomly selected rural and urban kebeles.

Mecha district is located $515 \mathrm{Km}$ from Addis Ababa North West of Ethiopia and $35 \mathrm{~km}$ from Bahir Dar. The district has 44 kebeles, 4 urban and 40 rural, with total population of 292,250, 147,700 male and 144,550 female (census 2007).

According to the district health office (DHO) report, there are 10 health centers and 26 health post and also there are 89 rural and 8 urban health extension workers currently working in the area. The estimated number of infants under one year is 10,506 in the year $2011 / 2012$.

\subsection{Study Design}

The study was community based cross-sectional quantitative type in which all mothers with infants aged 6-12 month from selected kebeles were interviewed with structured questionnaire.

\subsection{Study Period}

The period of the study was from Feb. to June 2012, when data collection held from March 20 to April 10, 2012.

\subsection{Source Population and Study Population}

The source population was all mothers who have 6-12 month of age infants in the District and study population were all mothers who have 6-12 month of age infants living in the randomly selected kebeles of the District.

\subsection{Sample Size Determination and Sampling Procedure}

The sample was determined using a single population proportion formula by using prevalence (P) of EBF 58.3\% from previous study in Amhara region [29] and assuming 95\% confidence level and $5 \%$ degree of precision. Then the sample size was calculated as:

$$
\mathrm{n}=\frac{\left(Z_{\alpha / 2}\right)^{2} p(1-p)}{d^{2}} x D
$$

Where:

$\mathrm{n}$ - Minimum sample size required

$\mathrm{p}$ - An estimate of the proportion of 6-12 month infants exclusively breast feed

d- Margin of error for sampling

$Z_{\alpha / 2}$ - The standard normal value at $(100 \%-\alpha)$ confidence level

$\mathrm{D}=$ Design effect $=2$

$$
\mathrm{n}=\frac{(1.96)^{2}(0.583)(0.417)}{(0.05)^{2}} X 2=748
$$


Considering $5 \%$ for non-response rate, the final sample size found to be 786

\section{Results}

Out of the 819 eligible mothers approached, all agreed to participate in the study which made the response rate $100 \%$. The total number of mothers enrolled is above 786, the minimum sample size calculated, due to cluster sampling technique.

\subsection{Socio-Demographic Characteristics}

Table 1. Socio-demographic Characteristics of mothers with their infants aged 6-12 months (n=819), Mecha district, West Gojjam Zone, North West Ethiopia, April 2012.

\begin{tabular}{|c|c|c|}
\hline Variable & Category & Number (\%) \\
\hline \multirow{2}{*}{ Residence } & Urban & $104(12.7)$ \\
\hline & Rural & $715(87.3)$ \\
\hline \multirow{2}{*}{ Religion of Mother } & Orthodox Christian & $789(96.3)$ \\
\hline & Others & $30(3.7)$ \\
\hline \multirow{5}{*}{ Age of mother(Years) } & 15-19 Years & $25(3.1)$ \\
\hline & 20-24 Years & $151(18.4)$ \\
\hline & 25-29 Years & $318(38.8)$ \\
\hline & 30-34 Years & $203(24.8)$ \\
\hline & 35 Years and above & $122(14.9)$ \\
\hline \multirow{2}{*}{ Age of infant(months) } & 6-9 Months & $535(65.3)$ \\
\hline & 10-12 Months & $284(34.7)$ \\
\hline \multirow{2}{*}{ Sex of infant } & Male & $431(52.6)$ \\
\hline & Female & $388(47.4)$ \\
\hline \multirow{2}{*}{$\begin{array}{l}\text { Number of children } \\
\text { currently having }\end{array}$} & 1-2 children & $280(34.2)$ \\
\hline & 3 and above & $539(65.8)$ \\
\hline \multirow{3}{*}{$\begin{array}{l}\text { Birth order of the index } \\
\text { infant }\end{array}$} & First & $126(15.4)$ \\
\hline & Second & $154(18.8)$ \\
\hline & Third and above & $539(65.8)$ \\
\hline \multirow{2}{*}{$\begin{array}{l}\text { Birth interval from } \\
\text { preceding birth }(n=693)\end{array}$} & Below 3 years & $256(36.9)$ \\
\hline & 3 years and above & $437(63.1)$ \\
\hline \multirow{3}{*}{ Marital status of mother } & Married (in union) & $740(90.4)$ \\
\hline & Single & $59(7.2)$ \\
\hline & Others & $20(2.4)$ \\
\hline \multirow{2}{*}{ Educational status of mother } & Uneducated & $682(83.3)$ \\
\hline & Educated & $137(16.7)$ \\
\hline \multirow{3}{*}{ Occupation of mother } & Farmer & $703(85.8)$ \\
\hline & Merchant & $73(8.9)$ \\
\hline & Other & $43(5.3)$ \\
\hline \multirow{2}{*}{$\begin{array}{l}\text { Educational status of } \\
\text { husband }(n=744)\end{array}$} & Uneducated & $583(78.40$ \\
\hline & Educated & $161(21.6)$ \\
\hline
\end{tabular}

Most study participants $715(87.3 \%)$ were from rural areas. Majority of these mothers $789(96.3 \%)$ were orthodox Christian followers. The mean age of mothers was $28.2(\mathrm{SD} \pm 5.1)$ years, which range $17-42$ years. The mean age of infants was $8.8(\mathrm{SD} \pm 1.9))$ months, which range 6-12 months. Nearly two third $(65.3 \%)$ of infants were in the age group 6-9 months. Male infants were 431(52.6\%) and male to female ratio was $1.11: 1$
About two third of the mothers 539(65.8\%) have 3 and above children. The mean number of children per mother for this study found to be $3.5(\mathrm{SD} \pm 1.9)$ which range 1-8 children. Regarding the birth order of the index infants, $126(15.4 \%)$ was first birth order. Among the multiparous mothers majority, 437(63.1\%) have 3 years and above birth interval from preceding birth.

Regarding marital status of mothers, 740(90.4\%) were married and in union, 59(7.2\%) were single. Many of the mothers 682(83.3\%) are uneducated and abut $583(78.4 \%)$ of their husbands are also uneducated. Majority of the mothers 703(85.8\%) were farmers (table 1).

\subsection{Factors Associated with Exclusive Breast Feeding}

In the bivariate analysis; residence of respondent, husband educational status, ANC visit, post natal counseling about infant feeding, maternal knowledge about $\mathrm{BF}$ and immediate $\mathrm{BF}$ initiation after birth showed association with EBF practice.

But in the step wise multivariate logistic regression, residence, ANC visit, post natal counseling about infant feeding, maternal knowledge about $\mathrm{BF}$ and immediate initiation of breast feeding after birth were the independent factors associated with exclusive breastfeeding practice whereas birth order of index infant and husband educational status were not associated with EBF practice of the mother.

Women who were from urban areas were 0.38 times less likely to exclusively breastfeed compared to rural mothers [AOR $(95 \%)=0.38(0.23-0.62)]$. Mothers who got three and above ANC visit were 1.7 times more likely to breastfeed their infants exclusively than those who did not get ANC visit [ AOR $(95 \%)=1.7(1.09-2.67]$. Those mothers who got PNC counseling on infant feeding were 2.27 times more likely to practice EBF than their counterparts [ AOR $(95 \%)=2.27(1.56-3.30)]$.

Mothers who initiated BF immediately after birth were 2.98 times more likely to practice EBF than those who did not initiate BF immediately after birth. Those mothers who have adequate knowledge on $\mathrm{BF}$ were 2.06 times more likely to exclusively breast feed their infants than those mothers who did not have adequate knowledge on breast feeding $[$ AOR $(95 \%)=2.06(1.47-2.88)]($ table 2$)$.

\section{Discussion}

In this study the prevalence of exclusive breastfeeding at 6-months age of infants was $47.13 \%$ (95\%CI: $43.73 \%$ $-50.56 \%$ ) and the median duration of exclusive breast feeding was five month (i.e. $\mathrm{IQR}=6-4=2$ ). More over $270(32.97 \%)$ mothers reported as they predominantly breast feed in the first 6 month age of infants(figure 1) which is lower than the national prevalence whereas the median duration was found to be 5 month which is slightly higher than the national median duration (4.2 months) and Amhara region (4.6 Months) reported by EDHS [11]. 
Table 2. Bivariate and Multivariate analysis showing Factors Associated with EBF Practice of mothers with their 6-12 month aged infants $(n=819)$, Mecha district, West Gojjam Zone, North West Ethiopia, April 2012.

\begin{tabular}{|c|c|c|c|c|}
\hline \multirow{2}{*}{ Variables } & \multicolumn{2}{|l|}{ EBF } & \multirow{2}{*}{$\begin{array}{l}\text { COR }(95 \% \\
\text { CI) }\end{array}$} & \multirow{2}{*}{$\begin{array}{l}\operatorname{AOR}(95 \% \\
\text { CI) }\end{array}$} \\
\hline & Yes $(\%)$ & No $(\%)$ & & \\
\hline \multicolumn{5}{|l|}{ Residence } \\
\hline Urban & $32(30.8)$ & $72(69.2)$ & $\begin{array}{l}0.45 \\
(0.29-0.71)^{* *}\end{array}$ & $\begin{array}{l}0.38 \\
(0.23-0.62) * *\end{array}$ \\
\hline Rural & $\begin{array}{l}354 \\
(49.5)\end{array}$ & $361(50.5)$ & 1 & 1 \\
\hline \multicolumn{5}{|c|}{ Husband Educational Status } \\
\hline Uneducated & $\begin{array}{l}291(49.9 \\
)\end{array}$ & $292(50.1)$ & 1 & \\
\hline Educated & 64 (39.8) & $97(60.2)$ & $\begin{array}{l}0.66 \\
(0.46-0.94)^{*}\end{array}$ & \\
\hline No Husband & $31(41.3)$ & $44(58.7)$ & $\begin{array}{l}0.71 \\
(0.43-1.15)\end{array}$ & \\
\hline \multicolumn{5}{|l|}{ ANC Visit } \\
\hline No ANC Visit & )$^{101(31.3}$ & $222(68.7)$ & 1 & 1 \\
\hline $\begin{array}{l}1-2 \text { ANC } \\
\text { Visit }\end{array}$ & $135(50)$ & $135(50)$ & $\begin{array}{l}0.19 \\
(1.57-3.07)^{* *}\end{array}$ & $\begin{array}{l}1.04 \\
(0.69-1.56)\end{array}$ \\
\hline $\begin{array}{l}3 \text { and above } \\
\text { visit }\end{array}$ & $150(66.4$ & $76(33.6)$ & $\begin{array}{l}4.34 \\
(3.02-6.23)^{* *}\end{array}$ & $\begin{array}{l}1.7 \\
(1.09-2.67)^{*}\end{array}$ \\
\hline \multicolumn{5}{|c|}{ PNC counseling on infant feeding } \\
\hline Yes & $\begin{array}{l}231(66.8 \\
)\end{array}$ & $115(33.2)$ & $\begin{array}{l}4.12 \\
(3.07-5.53)^{* *}\end{array}$ & $\begin{array}{l}2.27 \\
(1.56-3.30)^{* * *}\end{array}$ \\
\hline No & $\begin{array}{l}155(32.8 \\
)\end{array}$ & $318(67.2)$ & 1 & 1 \\
\hline \multicolumn{5}{|c|}{ BF Knowledge of mother } \\
\hline Not adequate & )$^{125(31.6}$ & $270(68.4)$ & 1 & 1 \\
\hline Adequate & $\begin{array}{l}261(61.6 \\
)\end{array}$ & $163(38.4)$ & $\begin{array}{l}3.46 \\
(2.59-4.62)^{* *}\end{array}$ & $\begin{array}{l}2.06 \\
(1.47-2.88)^{* *}\end{array}$ \\
\hline \multicolumn{5}{|c|}{ Immediate breast feeding initiation } \\
\hline No & $73(26.1)$ & 207(73.9) & 1 & 1 \\
\hline Yes & $297(60)$ & 198(40) & $\begin{array}{l}4.25 \\
(3.08-5.87)^{* *}\end{array}$ & $\begin{array}{l}2.98 \\
(2.11-4.22)^{* * *}\end{array}$ \\
\hline $\begin{array}{l}\text { Don't } \\
\text { remember }\end{array}$ & $16(36.4)$ & $28(63.6)$ & $\begin{array}{l}1.62 \\
(0.83-3.17)\end{array}$ & $\begin{array}{l}1.54 \\
(0.74-3.2)\end{array}$ \\
\hline
\end{tabular}

$*$ P-valve $<0.05, * *$ p-valve $<0.001$

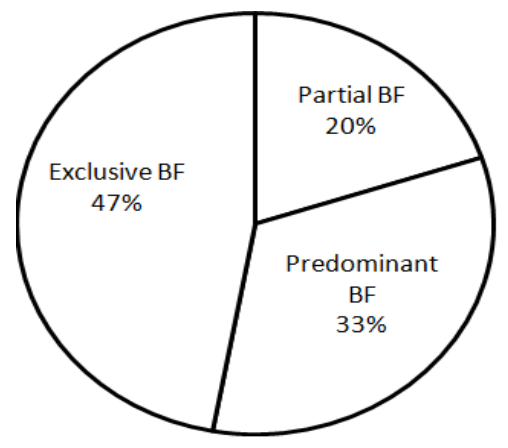

Figure 1. Breast feeding at 6 month among infants aged 6-12 month in Mecha district, West Gojjam, Amhara, North west Ethiopia, April 2012.

This study highlighted lower prevalence of EBF. It is still below the WHO recommendation which states as EBF is a cost-effective intervention in saving infants' live, when the coverage of $90 \%$ achieved $(8,9)$. In addition this prevalence is also lower than other study findings from India $57.1 \%$,
Nepal $60.5 \%$ Ghana 51.6\%, Pakistan $64.8 \%$ and Sirilanka 75\% [21], [25] , [30], [31], [32]. These results show variations of EBF prevalence between countries over time. In addition methodological differences for estimating the rate of EBF may also influence the results.

Previous study from Brazil revealed that greater maternal schooling favours the maintenance of EBF [33].But in this study maternal education was not associated with EBF practice. Previous study in Ethiopia also showed maternal education was not significantly associated with EBF practice [27].This may be due to that educated mothers may not have better knowledge regarding benefit of breast feeding than uneducated mothers.

A study from Nigeria showed female infants were more likely to be exclusively breastfeed than male infants [16]where as a study from Egypt [26] and Pakistan [21] revealed as male infants were more likely to exclusively breast feed than females counter parts. In our study sex of an infant is not associated with EBF. These controversies may be due to difference by socio-cultural factors which lead to gender difference in infant feeding practices and indicate a need for further studies, in order to better investigate the association.

Study from Ghana found that hospital/policlinic delivery was associated with higher likelihood of EBF practice [25].Another study from Tanzania also confirmed institutional delivery was positively associated with EBF [34].In contrast of these findings our study revealed institutional delivery was not associated with EBF practice. This may be due to short duration of health facility stay after delivery; or it might be difficult for health staff to give adequate information or for mothers to be receptive during when they are tired or experiencing pain.

This study revealed that mothers from urban areas were less likely to practice $\mathrm{EBF}[\mathrm{AOR}=0.38(0.23-0.62)]$ than rural mothers. This finding is consistent with a study done in Malaysia [24].This could be justified with that urban mothers have more chance for different job opportunities which limits time to stay with their infants which in turn can compromise EBF practice or it might be due to that urban mothers have more access for other infant feeding alternatives than rural mothers.

Among maternal health services ANC follow-up and PNC counselling on breast feeding are positively associated with EBF practice. Mothers who had three and more ANC visit were 1.7 time more likely to exclusively breast feed for 6 month as compared to those who have no ANC visit during pregnancy $[\mathrm{AOR}=1.7(1.09-2.67)]$.This is consistent with other study findings from Egypt [26]and Nigeria) [16].This might be the result from availability of policy, breastfeeding guidelines, and training for most staff on infant feeding in the era of PMTCT that contributes to have better knowledge and skills of counselling on EBF among health workers which in turn might have led to women getting adequate counselling about breast feeding during pregnancy.

Significant association was observed between PNC infant feeding counselling and the practice of EBF. The mothers 
who got PNC counselling on infant feeding are more likely to practice EBF as compared to those who did not get the counselling $[\mathrm{AOR}=2.27(1.56-3.30)]$. In line with this result, randomized controlled trial from Dhaka, Bangladesh revealed peer counselling from last trimester of pregnancy to postpartum period of mothers could significantly improve duration of EBF [35].

This could be that breast feeding counselling provided during postnatal period could positively influence mother's EBF practice since it is the most appropriate time for delivering key infant and young child feeding messages which enable to take immediate action.

Immediate initiation of breast feeding within one hour of birth has a positive influence on EBF practices. Infants who is initiated BF with in the first one hour of birth are 2.98 times more likely to exclusively breast feed than those who did not initiated BF immediately. This is in accordance with finding from Egypt which revealed infants who initiated BF immediately were 2.2 times more likely to exclusively breast feed than those who initiated BF after 24 hours [26].This may be linked with the fact that if breastfeeding is started late, it deprives babies stimulation and then limited breast secretion of the mothers that lead to the initiation of other feedings by mothers at early infant life.

Mothers who have adequate breast feeding knowledge were more likely to exclusively breast feed than mothers who have inadequate knowledge $[\mathrm{AOR}=2.06(1.47-2.88)]$.This clearly showed that the respondents who have adequate $\mathrm{BF}$ knowledge are 2.06 times more likely to practice EBF than those who have inadequate BF knowledge. This is in line with another finding in Tanzania that shows mothers who adequate knowledge of EBF were 5.4 times more likely to exclusively breast feed than mothers those who have not adequate knowledge [34].This could be that those who have not adequate knowledge on breast feeding tend to introduce other feedings in early age of infants.

\section{Conclusion}

Prevalence of EBF found in this study was lower than the WHO recommended level. It is also lower than the national figure reported by Ethiopian DHS.

Among different socio-demographic, health service, maternal, and infant related factors studied, only antenatal care (three and more visits), PNC infant feeding counseling, immediate breast feeding initiation after delivery and adequate knowledge on BF were the significant associated factors with higher chance of EBF practice whereas urban residence found to be associated with less exclusive breast feeding practice.

Based on the findings the following recommended actions for responsible bodies are forwarded

To Amhara Regional Health Bureau and NGOs

- Train health workers to ensure they have accurate and up-to- date information on infant feeding in general and breast feeding /EBF in particular which can help them to have specific knowledge \& skill required to educate and counsel mothers to improve EBF practice.

- Develop suitable IEC materials on BF to teach mothers at home and facility level.

To West Gojjam Zonal Health Department and district health Office

- Needs to expand and strengthen maternal health services specifically antenatal and postnatal care services in line with the existing health service expansion.

- Needs to deploy intensified promotional efforts on BF generally, EBF particularly by identifying urban areas as priority in line with the newly launched UHEP.

To HEWs and Other health care providers

- Strengthening and promote maternal health services specifically ANC and PNC services to address all eligible.

- Educate mothers on optimal breast feeding using appropriate IEC materials both at health facility and community level at large.

- Making BF counseling an important part of all maternal and child health services such as ANC,PNC,FP, vaccination and IMNCI to give health education and counseling for mothers that can improve their $\mathrm{BF}$ knowledge in turn to improve their EBF practice.

- Give intensive health education by targeting pregnant mothers on immediate $\mathrm{BF}$ initiation and counsel all mothers on infant feeding during PNC service which can substantially improve EBF practice.

To Researchers

- Further analytical studies, especially follow-up studies, are suggested to explore the actual levels of EBF and to further examine variables associated with the practice.

\section{References}

[1] World Health Organization. Learning from large-scale community-based program to improve breastfeeding practices. 2008

[2] World Health Organization. Mortality and burden of disease attributable to selected major risks. 2009.

[3] UNICEF. Tracking Progress On child And Maternal Nutrition: A survival and Development priority. 2009.

[4] Ethiopia Federal Ministry of Health. National Strategy for child survival Addiss Ababa2005.

[5] Hall J. Effective community-based interventions to improve exclusive breast feeding at four to six months in low- and low-middle-income countries: a systematic review of randomized controlled trials. J Hall / Midwifery 2011(27):Epub 502.

[6] Black R, Allen L, eta 1. Maternal and Child Under nutrition global and regional exposures and Study Group: Health consequences. Lancet. 2008;371( 9608 ):243-60.

[7] Amsalu S, Tigabu Z. Risk factors for severe acute malnutrition in children under the age of five: A case-control study EthiopJHealth Dev. 2008; 22(1):21-5. 
[8] Jones G, Steketee R, eta 1. How many child deaths can we prevent this year? Lancet. 2003;362:65-71.

[9] World Health Organization. Infant and young child feeding: Model Chapter for textbooks for medical students and allied health Professionals. Geneva2009.

[10] World Health Organization. Global Health Observation: Early initiation and exclusive breastfeeding Feb. 22/2012. Available

from :http://www.who.int/gho/child_health/prevention/breas tfeeding_text/en/index.html.

[11] CSA. Ethiopian DHS. Addis Ababa; Ethiopia2011.

[12] Teshome B, Getahun Z, Taye G. Magnitude and determinants of stunting in children underfive years of age in food surplus region of Ethiopia: The case of West Gojam Zone. Ethiop J Health Dev. 2009;23(2):98-106.

[13] Linkages. Academy for Educational Development: Facts for Feeding: Recommended Practices to Improve Infant Nutrition 2008

[14] US. Department of Health and Human Services :The Surgeon General's Call to Action to Support Breastfeeding Washington DC, USA 2011

[15] Kathryn A, Dorothy J, eta 1. Cultural Barriers to Exclusive Breastfeeding by Mothers in a Rural Area of Cameroon, Africa Journal of Midwifery \& Women's Health 2005(50):324-8.

[16] Agho, Kingsley E, Michael J, eta 1. Determinants of exclusive breastfeeding in Nigeria. BMC Pregnancy and Childbirth 2011;11(2).

[17] Mark W, . Early exclusive breastfeeding: still the cornerstone of child survival. Am J Clin Nutr. 2009(89):1281-2.

[18] Hoosen $M$, Nigel $C$, Ruth $M$, eta 1. Mother-to-child transmission of HIV-1 infection during exclusive breastfeeding in the first 6 months of life: an intervention cohort study. Lancet. 2007;369:1107-16.

[19] Foo L, Quek S, SA N, eta 1. Breastfeeding prevalence and practices among Singaporean Chinese, Malay and Indian mothers Health Prom International 2005;20(3):229-37.

[20] UNICEF. Regional Office for West and Central Africa: Protecting breastfeeding In west and central Africa. 2007.

[21] Aslam S, Sultan M, Akram F. Exclusive breast feeding; Duration at Norther Areas of Pakistan a hospital based study. Professional Med J 2010;17(2):286-90.

[22] Sapana P, Ameya H, eta 1. Prevalence of Exclusive Breast Feeding And Its Correlates In An Urban Slum In Western India. IeJSME 2009;3(2):14-8.
[23] Suneth B, Thilini C. Breastfeeding practices in a public health field practice area in Sri Lanka: a survival analysis International Breastfeeding Journal. 2007;2(13).

[24] Kok, Leong, Tan. Factors associated with exclusive breastfeeding among infants under six months of age in peninsular Malaysia. International Breastfeeding Journal 2011;6(2).

[25] Aidam B, Pe'rez R, eta 1. Factors associated with exclusive breastfeeding in Accra, Ghana. European Journal of Clinical Nutrition. 2005;59:789-96.

[26] Ghwass A, Ahmed D. Prevalence and Predictors of 6-Month Exclusive Breastfeeding in a Rural Area in Egypt Breastfeeding medicine 2011;6(4):191-6.

[27] Alemayehu T, Haidar J, Habte D. Determinants of exclusive breastfeeding practices in Ethiopia EthiopJHealth Dev 2009;23(1):12-8.

[28] Nekatebeb H, Guyon A, eta 1. Factors related to exclusive breast feeding and dietary diversity of complementary foods: A case study in Amhara region of Ethiopia. The FASEB Journal April 2010;Abstract 734.3.

[29] Fanosie F. Exclusive Breast Feeding Practices in Mida-Woremo District, Amhara Region, Ethiopia. Harar Bulletin of Health Sciences. 2010:Master Thesis.

[30] Sinhababu A, Dipta K, eta 1. Infant- and Young Child-feeding Practices in Bankura District, West Bengal, India. J HEALTH POPUL NUTR. 2010;28(3):294-9.

[31] Subba S, Chandrashekhar T, Binu V, Joshi H, eta 1. Infant feeding practices of mothers in an urban area in Nepal. Kathmandu Univ Med J (KUMJ). 2007;5(1):42-7.

[32] Suneth B, Thilini C, eta 1. Exclusive breastfeeding in Sri Lanka: problems of interpretation of reported rates: Commentary. International Breastfeeding Journal. 2009;4(14):doi:10.1186/746-4358-4-14.

[33] Venancio S, Monteiro C. Individual and contextual determinants of exclusive breast-feeding in Sa o Paulo, Brazil: a multilevel analysis Public Health Nutrition 2006;9(1):40-6: DOI: 10.1079/PHN2005760.

[34] Nkala, Msuya. Prevalence and predictors of exclusive breastfeeding among women in Kigoma region, Western Tanzania: a community based cross-sectional study. International Breastfeeding Journal. 2011;6(17): doi:10.1186/746-4358-6-17.

[35] Haider R, Ashworth A, Kabir L, eta 1, . Effect of community-based peer counsellors on exclusive breastfeeding practices in Dhaka, Bangladesh: a randomized controlled trial Lancet 2000. 